\title{
Noninvariant Hypersurfaces of a Nearly Trans-Sasakian Manifolds
}

\author{
Satya Prakash $\operatorname{Yadav}^{1}$ and Shyam Kishor ${ }^{2}$ \\ ${ }^{1}$ Department of Applied Science, Shambhunath Institute of Engineering and Technology, Allahabad 211 012, India \\ ${ }^{2}$ Department of Mathematics \& Astronomy, University of Lucknow, Lucknow 226 007, India
}

Correspondence should be addressed to Satya Prakash Yadav; satyamath23@rediffmail.com

Received 7 May 2014; Accepted 7 December 2014; Published 22 December 2014

Academic Editor: Bibhas R. Majhi

Copyright ( 2014 S. P. Yadav and S. Kishor. This is an open access article distributed under the Creative Commons Attribution License, which permits unrestricted use, distribution, and reproduction in any medium, provided the original work is properly cited.

The present paper focuses on the study of noninvariant hypersurfaces of a nearly trans-Sasakian manifold equipped with $(f, g, u, v, \lambda)$-structure. Initially some properties of this structure have been discussed. Further, the second fundamental forms of noninvariant hypersurfaces of nearly trans-Sasakian manifolds and nearly cosymplectic manifolds with $(f, g, u, v, \lambda)$-structure have been calculated provided $f$ is parallel. In addition, the eigenvalues of $f$ have been found and proved that a noninvariant hypersurface with $(f, g, u, v, \lambda)$-structure of nearly cosymplectic manifold with contact structure becomes totally geodesic. Finally the paper has been concluded by investigating the necessary condition for totally geodesic or totally umbilical noninvariant hypersurface with $(f, g, u, v, \lambda)$-structure of a nearly trans-Sasakian manifold.

\section{Introduction}

A hypersurface such that transform of a tangent vector of the hypersurface by the $(1,1)$ structure tensor field $\phi$ defining the almost contact structure is never tangent to the hypersurface was studied by Goldberg and Yano in 1970 [1]. Yano and Okumura [2] introduced $(f, g, u, v, \lambda)$-structure and termed it as a noninvariant hypersurface of almost contact metric manifold. In this paper, they showed that there always exists a $(f, g, u, v, \lambda)$-structure and gave the results that there does not exist an invariant hypersurface of a contact manifold. As a consequence, an invariant hypersurface of (almost) cosymplectic manifold is (almost) Kähler manifold. Further, they proved that there is no noninvariant hypersurfaces of a Sasakian manifold. In 1990, Chen [3] introduced slant submanifold which is a generalization of invariant and antiinvariant submanifold of almost Hermite manifold. Recently, Prasad [4] studied the noninvariant hypersurface of transSasakian manifold. In this paper, we study noninvariant hypersurface to the setting of nearly trans-Sasakian manifold.

\section{Preliminaries}

Let $\widetilde{M}$ be an almost contact metric manifold with almost contact metric structure $(\phi, \xi, \eta, g)$; that is, $\phi$ is $(1,1)$ tensor field, $\eta$ is 1 -form, and $g$ is a compatible Riemannian metric such that

$$
\begin{gathered}
\phi^{2}=-I+\eta \otimes \xi, \quad \eta(\xi)=1, \phi(\xi)=0, \eta \circ \phi=0, \\
g(\phi X, \phi Y)=g(X, Y)-\eta(X) \eta(Y), \\
g(X, \phi Y)=-g(\phi X, Y), \quad g(X, \xi)=\eta(X)
\end{gathered}
$$

for all $X, Y \in T \widetilde{M}$.

An almost contact metric manifold $\widetilde{M}$ is called a Sasakian manifold if there exists a killing vector field $\xi$ of unit length on $\widetilde{M}$ so that the tensor field $\phi$ of type $(1,1)$, defined by $\phi(X)=$ $-\nabla_{X} \xi$, satisfies the condition

$$
\left(\widetilde{\nabla}_{X} \phi\right) Y=g(X, Y) \xi-g(\xi, Y) X,
$$

for any pair of vector fields $X$ and $Y$ on $\widetilde{M}$. 
The notion of trans-Sasakian structure is given by Oubina [5]. In 1990, Blair and Oubina [6] find the condition

$$
\begin{aligned}
\left(\widetilde{\nabla}_{X} \phi\right) Y= & \alpha(g(X, Y) \xi-\eta(Y) X) \\
& +\beta(g(\phi X, Y) \xi-\eta(Y) \phi X),
\end{aligned}
$$

for all vector fields $X, Y$ on $\widetilde{M}$ and where $\alpha$ and $\beta$ are smooth functions. In this case we say that the trans-Sasakian structure is of type $(\alpha, \beta)$.

Gherghe introduced a nearly trans-Sasakian structure of type $(\alpha, \beta)$. An almost contact metric manifold $\widetilde{M}$ with almost contact metric structure $(\phi, \xi, \eta, g)$ is said to be a nearly transSasakian manifold [7] if

$$
\begin{aligned}
\left(\widetilde{\nabla}_{X} \phi\right) Y+\left(\widetilde{\nabla}_{Y} \phi\right) X \\
=\alpha(2 g(X, Y) \xi-\eta(Y) X-\eta(X) Y) \\
\quad-\beta(\eta(Y) \phi X+\eta(X) \phi Y)
\end{aligned}
$$

for all vector fields $X, Y$ on $\widetilde{M}$, where $\alpha$ and $\beta$ are smooth functions on $\widetilde{M}$ and $\widetilde{\nabla}$ is the operator of covariant differentiation with respect to $g$.

Moreover, a nearly trans-Sasakian structure of type $(\alpha, \beta)$ is nearly Sasakian or nearly Kenmotsu or nearly cosymplectic according to $\alpha=1, \beta=0$, or $\alpha=0, \beta=1$, or $\alpha=\beta=0$, respectively.

From (4), we have

$$
\widetilde{\nabla}_{X} \xi=-\alpha \phi X+\beta(X-\eta(X) \xi)-\phi\left(\widetilde{\nabla}_{\xi} \phi\right) X
$$

The Gauss and Weingarten formulae are given by

$$
\begin{gathered}
\widetilde{\nabla}_{X} Y=\nabla_{X} Y+\sigma(X, Y) \widehat{N} \\
\widetilde{\nabla}_{X} \widehat{N}=-A_{\widehat{N}} X
\end{gathered}
$$

for all $X, Y \in T M$, where $\widetilde{\nabla}$ and $\nabla$ are the Riemannian and induced Riemannian connections in $\widetilde{M}$ and $M$, respectively, and $\widehat{N}$ is the unit normal vector in the normal bundle $T^{\perp} M$. In this formula $\sigma$ is the second fundamental form on $M$ related to $A_{\widehat{N}}$ by

$$
\sigma(X, Y)=g\left(A_{\widehat{N}} X, Y\right)
$$

Let $M$ be a hypersurface of an almost contact metric manifold; then we define the following:

$$
\begin{gathered}
\phi X=f X+u(X) \widehat{N}, \\
\phi \widehat{N}=-U, \\
\xi=V+\lambda \widehat{N} ; \quad \lambda=\eta(\widehat{N}), \\
\eta(X)=v(X) .
\end{gathered}
$$

For $X \in T M$, we get an induced $(f, g, u, v, \lambda)$-structure $[2,8]$ on the noninvariant hypersurface satisfying

$$
\begin{gathered}
f^{2}=-I+u \otimes U+v \otimes V, \\
f U=-\lambda V, \quad f V=\lambda U, \\
u o f=\lambda v, \quad v o f=-\lambda u, \\
u(U)=1-\lambda^{2}, \quad u(V)=v(U)=0, \quad v(V)=1-\lambda^{2}, \\
g(f X, f Y)=g(X, Y)-u(X) u(Y)-v(X) v(Y), \\
g(X, f Y)=-g(f X, Y), \quad g(X, U)=u(X), \\
g(X, V)=v(X),
\end{gathered}
$$

for all $X, Y \in T M ; \lambda=\eta(\widehat{N})$.

Therefore we see that every transversal hypersurface of an almost contact Riemannian manifold also admits a $(f, g, u, v, \lambda)$-structure.

\section{Noninvariant Hypersurfaces with $(f, g, u, v, \lambda)$-Structure}

A noninvariant hypersurface of an almost contact manifold [1] is a hypersurface such that the transform of a tangent vector of the hypersurface by a linear transformation field $\phi$ of type $(1,1)$ acting in each tangent space $T_{P} M$ of $\widetilde{M}, P \in$ $\widetilde{M}$ defining the almost contact structure, is never tangent to the hypersurface. Let $X$ be a tangent vector of noninvariant hypersurface and then $\phi X$ will never be tangent to the hypersurface defined by (8).

A hypersurface of an almost contact manifold does not in general possess an almost complex structure. Goldberg and Yano [1] showed that there does not exist an invariant hypersurface of a contact manifold. This statement says that it is impossible to imbed a manifold as an invariant hypersurface of a contact space. It is well known that a hypersurface (real codimension 1) of an almost complex manifold admits an almost contact structure. However, this hypersurface clearly is not invariant, since the real codimension is 1; otherwise it admits an almost complex structure.

Consider noninvariant hypersurfaces of almost contact manifolds $\widetilde{M}$. These again admit almost complex structures but, in addition, there is a distinguished 1-form $\eta$ induced by the contact form of $\widetilde{M}$.

Lemma 1. Let $M$ be a noninvariant hypersurface with ( $f$, $g, u, v, \lambda)$-structure of a nearly trans-Sasakian manifold $\widetilde{M}$. Then

$$
\begin{aligned}
\left(\widetilde{\nabla}_{X} \phi\right) Y+\left(\widetilde{\nabla}_{Y} \phi\right) X \\
=\left(\left(\nabla_{X} u\right) Y+\left(\nabla_{Y} u\right) X+\sigma(X, f Y)+\sigma(Y, f X)\right) \widehat{N} \\
\quad+\left(\nabla_{X} f\right) Y+\left(\nabla_{Y} f\right) X+2 \sigma(X, Y) U \\
\quad-u(Y) A_{\widehat{N}} X-u(X) A_{\widehat{N}} Y,
\end{aligned}
$$




$$
\begin{gathered}
\left(\widetilde{\nabla}_{X} \eta\right) Y+\left(\widetilde{\nabla}_{Y} \eta\right) X=\left(\nabla_{X} v\right) Y+\left(\nabla_{Y} v\right) X-2 \lambda \sigma(X, Y) \\
\tilde{\nabla}_{X} \xi=\left(\nabla_{X} V-\lambda A_{\widehat{N}} X\right)+(\sigma(X, V)+X \lambda) \widehat{N},
\end{gathered}
$$

for all $X, Y \in T M$.

Proof. Consider

$$
\begin{aligned}
\left(\widetilde{\nabla}_{X} \phi\right) Y= & \left(\widetilde{\nabla}_{X} \phi Y\right)-\phi\left(\widetilde{\nabla}_{X} Y\right) \\
= & \nabla_{X} f Y+\sigma(X, f Y) \widehat{N}+u(Y)\left(-A_{\widehat{N}} X\right) \\
& +\left(\nabla_{X} u(Y)\right) \widehat{N} \\
& -f\left(\nabla_{X} Y\right)-u\left(\nabla_{X} Y\right) \widehat{N}+\sigma(X, Y) U \\
= & \left(\nabla_{X} f\right) Y-u(Y) A_{\widehat{N}} X+\sigma(X, Y) U \\
& +\left(\left(\nabla_{X} u\right) Y+\sigma(X, f Y)\right) \widehat{N}, \\
\left(\widetilde{\nabla}_{Y} \phi\right) X= & \left(\nabla_{Y} f\right) X-u(X) A_{\widehat{N}} Y+\sigma(X, Y) U \\
& +\left(\left(\nabla_{Y} u\right) X+\sigma(Y, f X)\right) \widehat{N},
\end{aligned}
$$

by using (6), (8), and (9). Then from (16) and (17), we have (13).

Next,

$$
\begin{aligned}
\left(\widetilde{\nabla}_{X} \eta\right) Y & =\widetilde{\nabla}_{X} \eta(Y)-\eta\left(\widetilde{\nabla}_{X} Y\right) \\
& =\nabla_{X}(v(Y))-v\left(\nabla_{X} Y\right)-\sigma(X, Y) \eta(\widehat{N}) .
\end{aligned}
$$

Therefore

$$
\left(\widetilde{\nabla}_{X} \eta\right) Y=\left(\nabla_{X} v\right) Y-\lambda \sigma(X, Y) .
$$

Similarly

$$
\left(\widetilde{\nabla}_{Y} \eta\right) X=\left(\nabla_{Y} v\right) X-\lambda \sigma(X, Y) .
$$

From (19) and (20), we get (14).

Further, consider

$$
\begin{aligned}
\widetilde{\nabla}_{X} \xi & =\nabla_{X} \xi+\sigma(X, \xi) \widehat{N} \\
& =\nabla_{X} V+\nabla_{X} \lambda \widehat{N}+\sigma(X, V) \widehat{N} \\
& =\nabla_{X} V+\lambda \nabla_{X} \widehat{N}+(X \lambda) \widehat{N}+\sigma(X, V) \widehat{N}
\end{aligned}
$$

which proves (15).

Proposition 2. Let $M$ be a noninvariant hypersurface with $(f, g, u, v, \lambda)$-structure of a nearly trans-Sasakian manifold $\widetilde{M}$, and then

$$
\begin{gathered}
\sigma(X, V)=-\alpha u(X)-\beta \lambda v(X)-u\left(\widetilde{\nabla}_{\xi} \phi\right) X-X \lambda, \\
\nabla_{X} V=\lambda A_{\widehat{N}} X-\alpha f(X)+\beta(X-v(X) V)-f\left(\widetilde{\nabla}_{\xi} \phi\right) X
\end{gathered}
$$

for all $X \in T M$.
Proof. From (5) and (15), we have

$$
\begin{aligned}
\left(\nabla_{X} V-\lambda A_{\widehat{N}} X\right)+(\sigma(X, V)+X \lambda) \widehat{N} \\
=-\alpha f X-\alpha u(X) \widetilde{N}+\beta X-\beta \lambda v(X) \widehat{N} \\
\quad-\beta v(X) V-f\left(\left(\widetilde{\nabla}_{\xi} \phi\right) X\right)-u\left(\left(\widetilde{\nabla}_{\xi} \phi\right) X\right) \widehat{N} .
\end{aligned}
$$

Equating tangential and normal parts, we have

$$
\begin{gathered}
\sigma(X, V)=-\alpha u(X)-\beta \lambda v(X)-u\left(\widetilde{\nabla}_{\xi} \phi\right) X-X \lambda, \\
\nabla_{X} V=-\alpha f(X)+\beta X-\beta v(X) V-f\left(\widetilde{\nabla}_{\xi} \phi\right) X+\lambda A_{\widehat{N}} X .
\end{gathered}
$$

Now we find some results on totally geodesic noninvariant hypersurfaces.

Theorem 3. Let $M$ be a totally umbilical noninvariant hypersurface with $(f, g, u, v, \lambda)$-structure of a nearly trans-Sasakian manifold. Then it is totally geodesic if

$$
\alpha u(X)+\beta \lambda v(X)+u\left(\left(\widetilde{\nabla}_{\xi} \phi\right) X\right)+X \lambda=0 .
$$

Also, if nearly trans-Sasakian manifold admits contact structure, then

$$
\frac{1}{\lambda} \alpha u+\beta v+d(\log \lambda)=0 .
$$

Proof. Consider

$$
\begin{aligned}
\widetilde{\nabla}_{X} \xi & =\nabla_{X} \xi+\sigma(X, \xi) \widehat{N} \\
& =\nabla_{X} V+\nabla_{X} \lambda \widehat{N}+\sigma(X, V) \widehat{N} \\
& =\nabla_{X} V+\lambda \nabla_{X} \widehat{N}+(X \lambda) \widehat{N}+\sigma(X, V) \widehat{N} .
\end{aligned}
$$

Then, we have

$$
\widetilde{\nabla}_{X} \xi=\left(\nabla_{X} V-\lambda A_{\widehat{N}} X\right)+(\sigma(X, V)+X \lambda) \widehat{N} .
$$

From (5) and (28), we calculate

$$
\begin{aligned}
\left(\nabla_{X} V-\lambda A_{\widehat{N}} X\right)+(\sigma(X, V)+X \lambda) \widehat{N} \\
=-\alpha f X-\alpha u(X) \widetilde{N}+\beta X-\beta v(X) V \\
\quad-\beta \lambda v(X) \widehat{N}-f\left(\left(\widetilde{\nabla}_{\xi} \phi\right) X\right)-u\left(\left(\widetilde{\nabla}_{\xi} \phi\right) X\right) \widehat{N} .
\end{aligned}
$$

Equating normal part, we have

$$
\sigma(X, V)=-\alpha u(X)-\beta \lambda v(X)-u\left(\widetilde{\nabla}_{\xi} \phi\right) X-X \lambda .
$$

If $M$ is totally umbilical, then $A_{\widehat{N}}=\zeta I$, where $\zeta$ is Kählerian metric [9] and we know the relation of $\sigma$ on $M$ related to $A_{\widehat{N}}$ by

$$
\sigma(X, Y)=g\left(A_{\widehat{N}} X, Y\right)=g(\zeta X, Y)=\zeta g(X, Y) .
$$


Therefore $\sigma(X, V)=\zeta g(X, V)=\zeta v(X)$, and then (30) gives

$$
\alpha u(X)+\beta \lambda v(X)+u\left(\widetilde{\nabla}_{\xi} \phi\right) X+X \lambda+\zeta v(X)=0 .
$$

If $M$ is totally geodesic, that is, $\zeta=0$, then (32) gives (25). If nearly trans-Sasakian manifold has contact structure, then from (25) we have (26).

Theorem 4. Let $M$ be a noninvariant hypersurface with ( $f$, $g, u, v, \lambda)$-structure of a nearly trans-Sasakian manifold. If $f$ is parallel, then one has

$$
\begin{aligned}
\sigma(X, Y)= & \frac{\alpha}{3\left(1-\lambda^{2}\right)}(u(Y) v(X)+u(X) v(Y)) \\
& +\frac{\beta \lambda}{\left(1-\lambda^{2}\right)} v(X) v(Y)+\frac{\mu}{3\left(1-\lambda^{2}\right)^{2}} u(X) u(Y),
\end{aligned}
$$

where $\mu=\sigma(U, U)=g\left(A_{\widehat{N}} U, U\right)$ and $f$ is a tensor field of type $(1,1)$. Also, $M$ is totally geodesic if

$$
\frac{4}{3} \alpha u(X)+2 \beta \lambda v(X)+u\left(\widetilde{\nabla}_{\xi} \phi\right) X+X \lambda=0 .
$$

Proof. From (4) and (13), we have easily found the relation

$$
\begin{aligned}
\left(\nabla_{X} f\right) Y+\left(\nabla_{Y} f\right) X & \\
= & \alpha(2 g(X, Y) V-v(Y) X-v(X) Y)+2 \sigma(X, Y) U \\
& -\beta(v(Y) f X+v(X) f Y)+u(Y) A_{\widehat{N}} X-u(X) A_{\widehat{N}} Y .
\end{aligned}
$$

Since $f$ is parallel then (35) reduces to

$$
\begin{aligned}
2 \sigma(X, Y) U= & \alpha(v(Y) X+v(X) Y-2 g(X, Y) V) \\
& -u(Y) A_{\widehat{N}} X \\
& -u(X) A_{\widehat{N}} Y+\beta(v(Y) f X+v(X) f Y) .
\end{aligned}
$$

Applying $u$ both sides, we obtain

$$
\begin{aligned}
2(1 & \left.-\lambda^{2}\right) \sigma(X, Y) \\
= & \alpha u(Y) v(X)+\alpha u(X) v(Y)+2 \beta \lambda v(X) v(Y) \\
& -u(Y) u\left(A_{\widehat{N}} X\right)-u(X) u\left(A_{\widehat{N}} Y\right) .
\end{aligned}
$$

In view of (37), we have

$$
u\left(A_{\widehat{N}} X\right)=\frac{\alpha}{3} v(X)-\frac{\mu}{3\left(1-\lambda^{3}\right)} u(X),
$$

where $\mu=\sigma(U, U)$.

In a similar way we have

$$
u\left(A_{\widehat{N}} Y\right)=\frac{\alpha}{3} v(Y)-\frac{\mu}{3\left(1-\lambda^{3}\right)} u(Y)
$$

Using (37)-(39), we have the result (33).
Next, from (30) and (33), we have

$$
\frac{4}{3} \alpha u(X)+2 \beta \lambda v(X)+u\left(\widetilde{\nabla}_{\xi} \phi\right) X+X \lambda=0 .
$$

If nearly trans-Sasakian with contact structure, then

$$
\frac{4}{3} \alpha u(X)+2 \beta \lambda v(X)+X \lambda=0
$$

which implies

$$
\frac{4}{\lambda} \alpha u+6 \beta \lambda v+3 d(\log \lambda)=0
$$

As a consequence, we have the following.

Corollary 5. Let $M$ be a noninvariant hypersurface with ( $f$, $g, u, v, \lambda)$-structure of a nearly cosymplectic manifold. If $f$ is parallel, then one has

$$
\sigma(X, Y)=\frac{\mu}{3\left(1-\lambda^{2}\right)^{2}} u(X) u(Y), \quad \text { where } \mu=\sigma(U, U) \text {. }
$$

Theorem 6. Let $M$ be a noninvariant hypersurface with $(f, g, u, v, \lambda)$-structure of a nearly trans-Sasakian manifold. If a vector field $U$ is parallel, then one has

$$
\alpha \lambda X+\beta \lambda f X+f A_{\widehat{N}} X+\left(\nabla_{\widehat{N}} f\right) X=0 .
$$

Consequently, if $M$ is totally geodesic and $\nabla_{\widehat{N}} f=0$ for all $X$, then eigenvalue of $f$ is $(-\alpha / \beta)$.

Proof. Consider

$$
\begin{aligned}
\left(\widetilde{\nabla}_{X} \phi\right) \widehat{N} & =\widetilde{\nabla}_{X} \phi \widehat{N}-\phi \widetilde{\nabla}_{X} \widehat{N} \\
& =-\widetilde{\nabla}_{X} U-\phi\left(-A_{\widehat{N}} X\right) \\
& =-\nabla_{X} U-\sigma(X, U) \widehat{N}+f\left(A_{\widehat{N}} X\right)+u\left(A_{\widehat{N}} X\right) \widehat{N} .
\end{aligned}
$$

Therefore

$$
\left(\widetilde{\nabla}_{X} \phi\right) \widehat{N}=-\nabla_{X} U+f\left(A_{\widehat{N}} X\right) .
$$

From (4), we have

$$
\begin{aligned}
\left(\widetilde{\nabla}_{X} \phi\right) Y+\left(\widetilde{\nabla}_{Y} \phi\right) X \\
=\alpha\{2 g(X, Y) V-\eta(Y) X-\eta(X) Y\} \\
\quad-\beta\{\eta(Y) f X+\eta(X) f Y\} \\
\quad+\{2 \alpha \lambda g(X, Y)-\beta \eta(Y) u(X)-\beta \eta(X) u(Y)\} \widehat{N} .
\end{aligned}
$$

From (47), we have

$$
\begin{aligned}
\left(\widetilde{\nabla}_{X} \phi\right) \widehat{N}+\left(\widetilde{\nabla}_{\widehat{N}} \phi\right) X \\
=-\alpha \lambda X-\alpha v(X) \widehat{N}-\beta \lambda f X \\
\quad-\beta v(X) f \widehat{N}-\{\beta \lambda u(X)+\beta v(X) u(\widehat{N})\} \widehat{N} .
\end{aligned}
$$


Then from (17) we have

$$
\begin{aligned}
\left(\widetilde{\nabla}_{\widehat{N}} \phi\right) X= & \left(\nabla_{\widehat{N}} f\right) X-u(X) A_{\widehat{N}} \widehat{N} \\
& +\left\{\left(\nabla_{\widehat{N}} u\right) X+\sigma(\widehat{N}, f X)\right\} \widehat{N} .
\end{aligned}
$$

Using (46), (48), and (49), we get

$$
\begin{aligned}
-\nabla_{X} U & +f\left(A_{\widehat{N}} X\right)+\left(\nabla_{\widehat{N}} f\right) X \\
= & u(X) A_{\widehat{N}} \widehat{N}-\left\{\left(\nabla_{\widehat{N}} u\right) X-\sigma(\widehat{N}, f X)\right\} \widehat{N} \\
& -\alpha \lambda X-\alpha v(X) \widehat{N}-\beta \lambda f X-\beta v(X) f \widehat{N} \\
& -\{\beta \lambda u(X)+\beta v(X) u(\widehat{N})\} \widehat{N} .
\end{aligned}
$$

Now equating tangential part, we have

$$
\nabla_{X} U=\alpha \lambda X+\beta \lambda f X+f\left(A_{\widehat{N}} X\right)+\left(\nabla_{\widehat{N}} f\right) X .
$$

If $U$ is parallel then (51) implies

$$
\alpha \lambda X+\beta \lambda f X+f\left(A_{\widehat{N}} X\right)+\left(\nabla_{\widehat{N}} f\right) X=0 .
$$

If $M$ is totally geodesic then $\zeta=0$; that is, $A_{\widehat{N}}=0$.

Therefore

$$
\alpha \lambda X+\beta \lambda f X+\left(\nabla_{\widehat{N}} f\right) X=0 .
$$

If $\nabla_{\widehat{N}} f=0$, then $\alpha \lambda X+\beta \lambda f X=0$, which implies $f X=$ $-(\alpha / \beta) X$.

Hence eigenvalue of $f$ is $(-\alpha / \beta)$.

Theorem 7. If $V$ is parallel vector field in noninvariant hypersurface $M$ with $(f, g, u, v, \lambda)$-structure of a nearly cosymplectic manifold $\widetilde{M}$ admitting contact structure, then

$$
\sigma(X, Y)=0 ; \quad \text { that is, } M \text { is totally geodesic. }
$$

Proof. We know that, for a nearly cosymplectic manifold $\widetilde{M}$,

$$
\widetilde{\nabla}_{X} \xi=-\phi\left(\widetilde{\nabla}_{\xi} \phi\right) X
$$

From (28) and (55), we have

$$
-\phi\left(\widetilde{\nabla}_{\xi} \phi\right) X=\left(\nabla_{X} V-\lambda A_{\widehat{N}} X\right)+(\sigma(X, V)+X \lambda) \widehat{N} .
$$

Equating tangential part, we have

$$
\nabla_{X} V=-f\left(\widetilde{\nabla}_{\xi} \phi\right) X+\lambda A_{\widehat{N}} X
$$

Since nearly cosymplectic manifold admits contact structure, then $\nabla_{X} V=\lambda A_{\widehat{N}} X$.

If $V$ is parallel, then $\lambda A_{\widehat{N}} X=0 \forall X \in T M$.

Since $\lambda \neq 0$, therefore $A_{\widehat{N}} X=0 \forall X \in T M$, which gives $g\left(A_{\widehat{N}} X, Y\right)=\sigma(X, Y)=0 \forall X \in T M$, which implies $M$ is totally geodesic.

Proposition 2 also enables us to deduce the above result by substituting $\alpha=0, \beta=0$ for a nearly cosymplectic manifold admitting contact structure (i.e., $\widetilde{\nabla}_{\xi} \phi=0$ ) provided $V$ is parallel.

\section{Conflict of Interests}

The authors declare that there is no conflict of interests regarding to the publication of this paper.

\section{Acknowledgments}

The authors would like to thank the anonymous reviewer for his valuable comments and suggestions to improve the quality of the paper. They also thank Dr. Rajendra Prasad for his help.

\section{References}

[1] S. I. Goldberg and K. Yano, "Noninvariant hypersurfaces of almost contact manifolds," Journal of the Mathematical Society of Japan, vol. 22, no. 1, pp. 25-34, 1970.

[2] K. Yano and M. Okumura, "On $(F, g, u, v, \lambda)$-structures," Kodai Mathematical Seminar Reports, vol. 22, pp. 401-423, 1970.

[3] B. Y. Chen, Geometry of Slant Submanifolds, Katholieke Universiteit Leuven, Leuven, Belgium, 1990.

[4] R. Prasad, "On non-invariant hypersurfaces of trans-Sasakian manifolds," Bulletin of the Calcutta Mathematical Society, vol. 99, no. 5, pp. 501-510, 2007.

[5] J. A. Oubina, "New classes of almost contact metric structures," Publicationes Mathematicae Debrecen, vol. 32, no. 3-4, pp. 187193, 1985.

[6] D. E. Blair and J. A. Oubina, "Conformal and related changes of metric on the product of two almost contact metric manifolds," Publicacions Matemàtiques, vol. 34, no. 1, pp. 199-207, 1990.

[7] C. Gherghe, "Harmonicity on nearly trans-Sasaki manifolds," Demonstratio Mathematica, vol. 33, no. 1, pp. 151-157, 2000.

[8] D. E. Blair, G. D. Ludden, and K. Yano, "Induced structures on submanifolds," Kodai Mathematical Seminar Reports, vol. 22, no. 2, pp. 188-198, 1970.

[9] S. I. Goldberg, "Conformal transformations of Kaehler manifolds," Bulletin of the American Mathematical Society, vol. 66, pp. 54-58, 1960. 


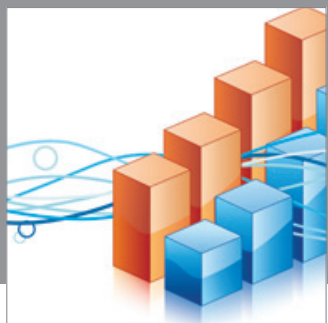

Advances in

Operations Research

mansans

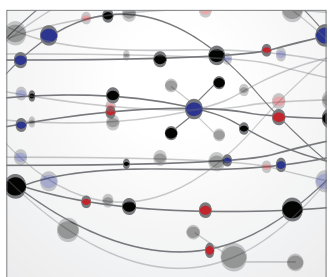

The Scientific World Journal
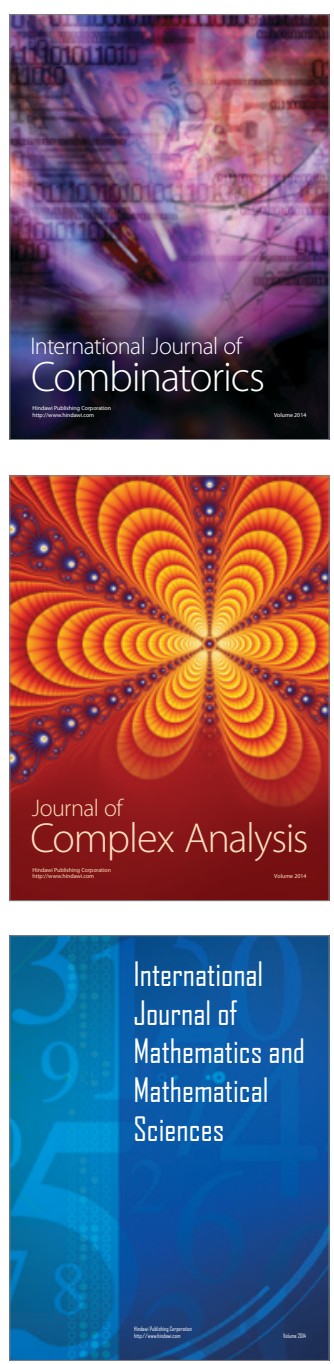
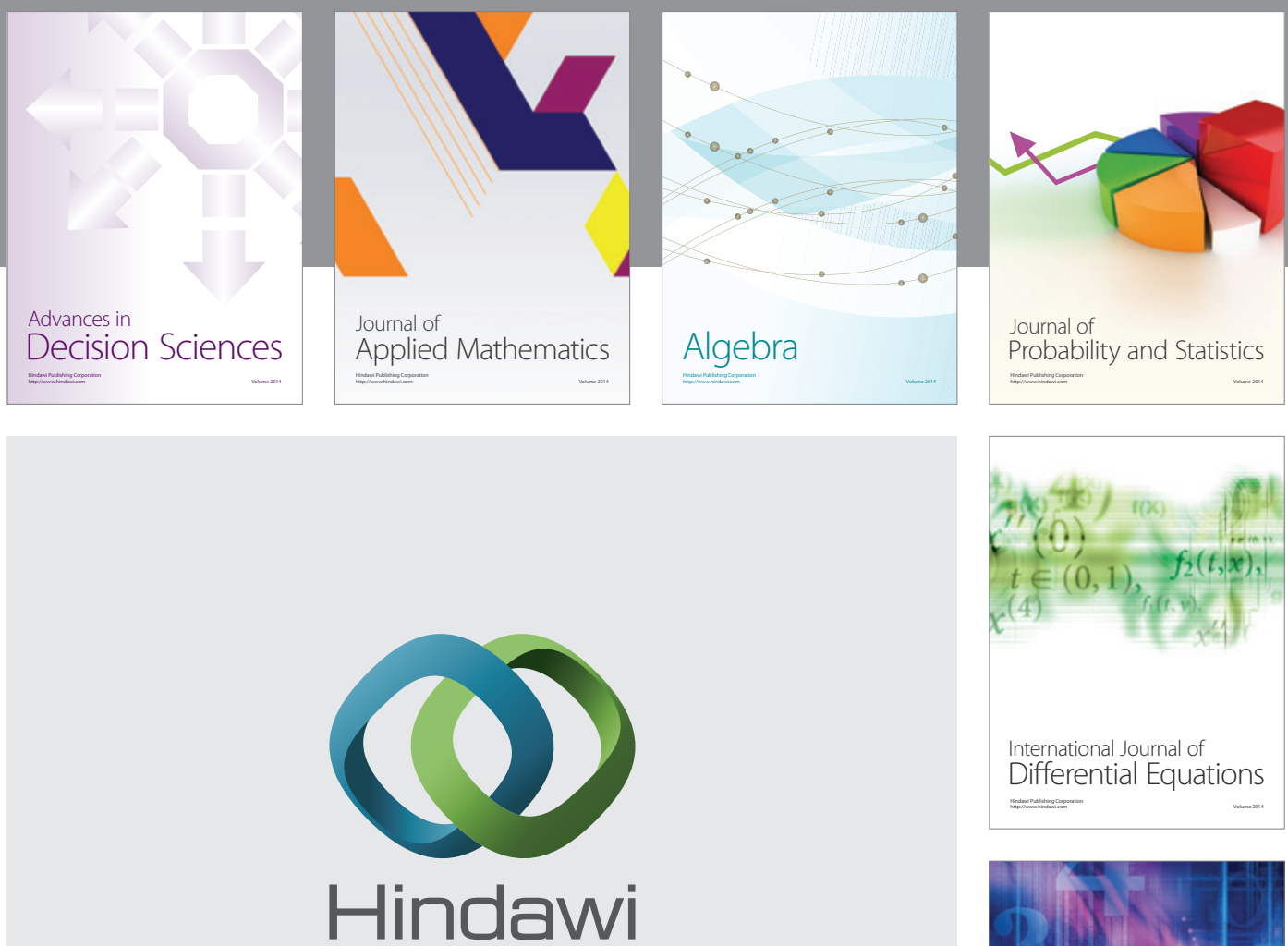

Submit your manuscripts at http://www.hindawi.com
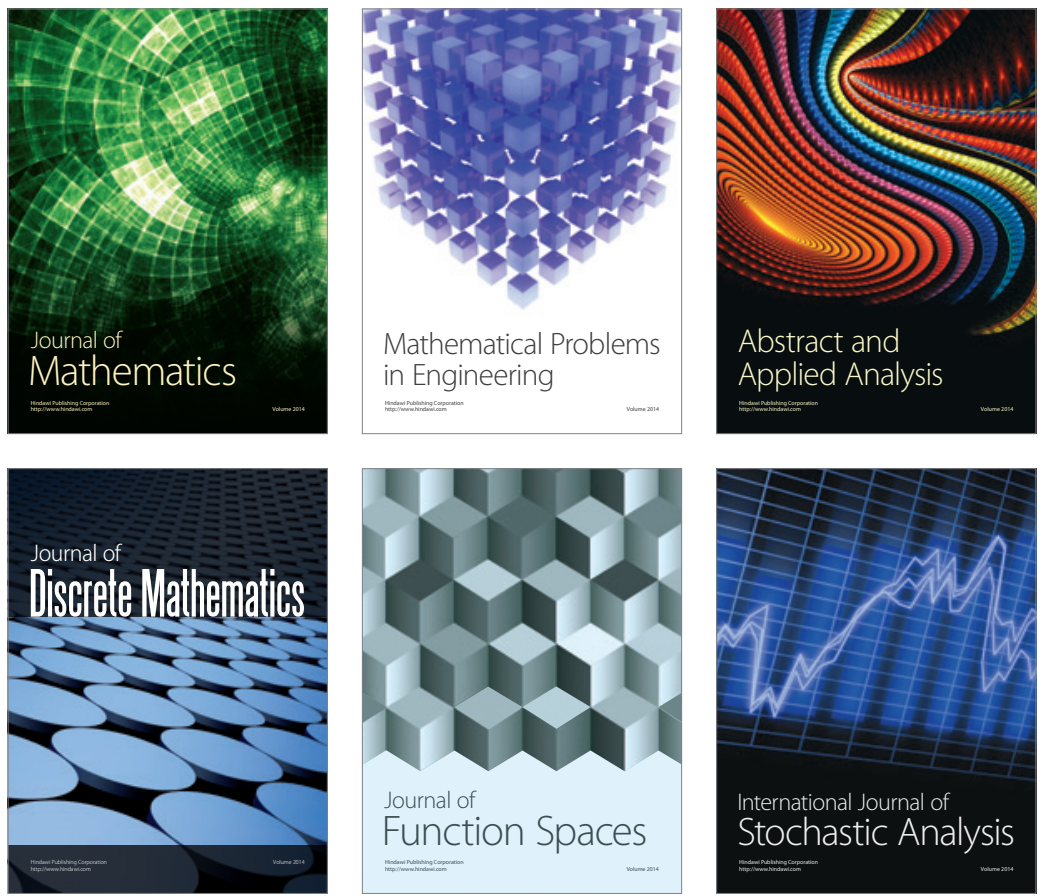

Journal of

Function Spaces

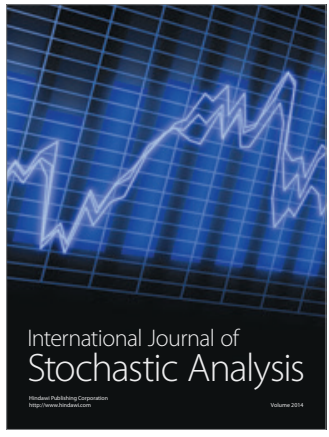

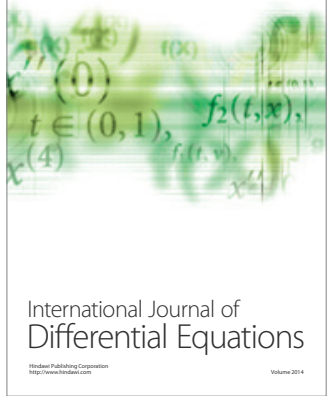
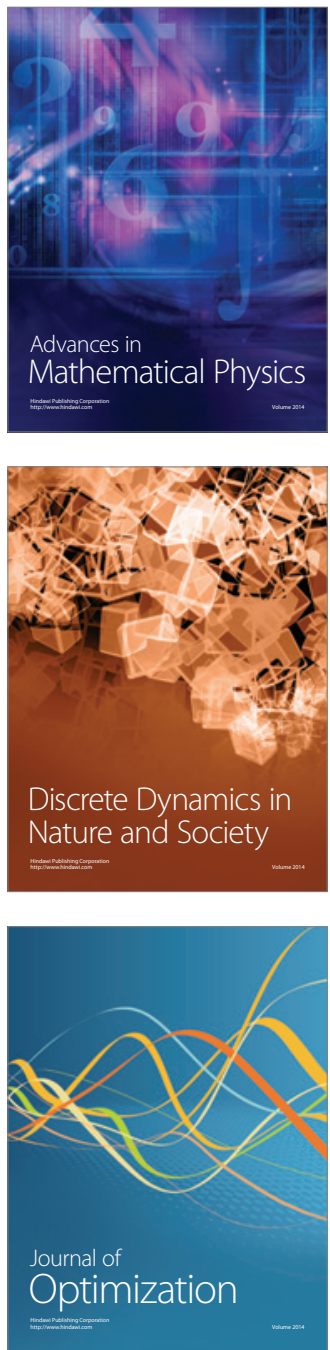